\title{
A Novel Phantom-Less Spatial and Temporal Ultrasound Calibration Method
}

\author{
Ali Khamene and Frank Sauer \\ Imaging and Visualization Dept., \\ Siemens Corporate Research, \\ 755 College Road East, Princeton NJ 08540, USA \\ $<$ first name>.<last name>Qscr.siemens.com
}

\begin{abstract}
This paper introduces a novel method for ultrasound calibration for both spatial and temporal parameters. The main advantage of this method is that it does not require a phantom, which is usually expensive to fabricate. Furthermore, the method does not require extensive image processing. For spatial calibration, we solve an optimization problem established by a set of equations that relate the orientations of a line (i.e., calibration pointer) to the intersection points appearing in the ultrasound image. The line orientation is provided through calibration of both ends of the calibration pointer. Temporal calibration is achieved by processing of the captured pointer orientations and the corresponding image positions of intersection along with the timing information. The effectiveness of the unified method for both spatial and temporal calibration is apparent from the quality of the $3 \mathrm{D}$ reconstructions of a known object.
\end{abstract}

\section{Introduction and Background}

Ultrasound imaging systems are being widely used in many interventional and radiation therapy applications. In these applications, ultrasound probe is usually instrumented with a tracking sensor (either magnetic or optical) or an articulated arm providing the estimates of position and orientation (i.e., pose) of the probe at all times. A calibration process has to establish the transformation from an anatomical location appearing in the ultrasound image to the coordinate system established by the external tracking device. This is referred to as spatial calibration. Furthermore, the exact timing at which the ultrasound image is captured has to be synchronized to the positional information read from the external tracking system. This is referred to as temporal calibration. The cumulative accuracy of tracking system and calibration parameters dictates the fidelity of the overall system to quantify anatomical locations in the desired coordinate system. A tracked ultrasound imaging system can be used to bring anatomical location and surgical instruments or the iso-center of a radiation beam into the same coordinate system 24. Another application is to compound 3D ultrasound volumes for visualization and quantification [8]. 
Ultrasound spatial calibration methods have widely been investigated in the literature. There are varieties of geometrical phantoms proposed in the literature mainly to facilitate the calibration procedure. Phantoms with sparse set of wires are proposed in [13. In these procedures, intersection points of the wires are being imaged and are related to the known 3D position of the same point in the phantom. In 9], the proposed phantom has a special shape with known control points, which is then used to relate the local coordinate system of the phantom to that of the tracking system. In 2], a Z-shaped based phantom is used reduce the scan time and to facilitate the process of establishing correspondences between features in the ultrasound image and that in the phantom. In [6], a more sophisticated phantom is used, which combines strings and fiducials.

The main problem in using a phantom is that first the manufacturing of an accurate phantom is expensive and second no matter how accurate the phantom is, its position (or local coordinate system) has to be determined in the tracking system either by attaching another sensor to the phantom at the some exact position or to use control points on the phantom as beacons for registration. In either case the accuracy of the phantom is bound to the accuracy of the tracking system. In [7, authors propose a single-wall phantom that is very easy to use and does not require establishing any correspondences. The equation of the wall has to be determined in the tracking device coordinate system. Furthermore, a special holder has to be used for scanning procedure. The number of images required for this calibration process is rather high. In [5], authors propose a calibration method that does not require a phantom. A calibrated pointer tip is placed into the ultrasound beam. The traces of the pointer tip in the image and the threedimensional locations acquired with the tracking system are collected and used to compute calibration parameters. The drawback of this approach is that it is very hard to pinpoint the exact position at which the pointer enters the plane. This is mostly because of the beam width of the ultrasound imaging system. This effect makes the whole process inaccurate in determining the calibration parameters, specifically regarding translation in the direction perpendicular to the ultrasound plane.

Our proposed method is inspired by the method described in [5]. However, we address the shortcomings of the previous method. Furthermore, we add an essential piece for estimating the temporal lead/lag between tracking information and ultrasound frame. In contrast to [5], we use a line pointer instead of a "point pointer". I.e., we formulate the problem, in a way that we only require the direction of a tracked pointer in $3 \mathrm{D}$ and not the location of pointer tip. Therefore, as long as the pointer intersects the ultrasound plane, we are acquiring valid information for the calibration process.

\section{Ultrasound Calibration}

Conventional ultrasound imaging systems provide real-time two dimensional array of pixels (i.e., $I(u, v)$ ) refreshed at the frequency of $f_{u s}$. Real-time optical, magnetic, or mechanical tracking devices are used to map ultrasound images 
taken from various positions into a global coordinate system. Let us denote the world coordinate system of the tracking device as $\mathbf{W}$, and the coordinate system of the sensor $i$ as $\mathbf{S}_{i}$. Tracking device provides homogenous transformation matrices ${ }^{W} \mathbf{T}_{S_{i}}$ relating the sensor coordinate $\mathbf{S}_{i}$ to the world coordinate system $\mathbf{W}$ with certain refresh frequency of $f_{t r}$. Spatial calibration parameters can be presented as the combination of a homogenous scaling matrix $\mathbf{T}_{s}$ and a transformation matrix $\mathbf{T}_{c}$. In order to map any point in the ultrasound image plane (i.e., $P_{u}=[u, v, 0,1]^{\top}$ ) to the world coordinate system the following matrix multiplication chain has to be computed:

$$
P_{w}^{t}={ }^{W} \mathbf{T}_{S_{0}}^{t} \mathbf{T}_{c} \mathbf{T}_{s} P_{u}^{t}
$$

where the scaling factors in horizontal and vertical directions are built into $\mathbf{T}_{s}=$ $\operatorname{diag}\left(s_{x}, s_{y}, 0,1\right)$, and it is assumed that the ultrasound probe is instrumented with the tracking sensor number 0. Furthermore, time dependency is denoted by $t$. Sampling interval is usually dictated by $\delta t=\frac{1}{f_{u s}}$. Temporal calibration (synchronization) process is to infer $\mathbf{T}_{S_{0}}$ at time $n \delta t$ ( $n$ is an integer) from the samples available at the time intervals of $\frac{1}{f_{t r}}$. The synchronization is dominated by inherent delays, which exist in both ultrasound image formation and tracking. Therefore, the temporal calibration parameter is a single number $\tau$ representing the delay, which is usually much larger than the sampling interval of either ultrasound or tracker. For the case where the sampling frequency is identical (i.e., $f_{u s}=f_{t r}$ ) perfect synchronization can be achieved. However, in the case where the sampling frequencies are not identical, the time discrepancy varies in the range of $\tau \pm \min \left(\frac{1}{f_{u s}}, \frac{1}{f_{t r}}\right)$.

\subsection{Phantom-Less Calibration}

A calibration procedure that does not require a phantom is proposed in [5]. In this approach, a secondary tracked coordinate system (i.e., $\mathbf{S}_{1}$ ) is considered. The pointer is in fact a known fixed coordinate $P_{p}$ within such a coordinate system. By carefully placing the pointer tip into the ultrasound beam and recording both the trace of the point in the image $P_{u}^{i}$ and the coordinate transformation of the sensor attached to the pointer, one can establish the following relationship:

$$
{ }^{W} \mathbf{T}_{S_{1}}^{i} P_{p}={ }^{W} \mathbf{T}_{S_{0}}^{i} \mathbf{T}_{c} \mathbf{T}_{s} P_{u}^{i}
$$

Therefore, the spatial calibration procedure can be thought of as an optimization process finding eight parameters (i.e., six for translation and rotation and two for the scaling) lumped into matrices $\mathbf{T}_{c}$ and $\mathbf{T}_{s}$, as follows:

$$
\left\{\widetilde{\mathbf{T}_{\mathbf{c}}}, \widetilde{\mathbf{T}_{s}}\right\}=\arg \min _{\left[\mathbf{T}_{c}, \mathbf{T}_{s}\right]} \sum_{i}\left\|\left[{ }^{W} \mathbf{T}_{S_{1}}^{i} P_{p}-{ }^{W} \mathbf{T}_{S_{0}}^{i} \mathbf{T}_{c} \mathbf{T}_{s} P_{u}^{i}\right]_{3}\right\|^{2}
$$

where operator $[.]_{3}$ converts homogenous to Euclidian coordinates by dropping the fourth element, and $\|$.$\| represents Euclidian norm. The main problem with$ this approach is that it is very hard to make sure that the pointer tip is exactly in the ultrasound plane. The trace of the pointer observed within the ultrasound 


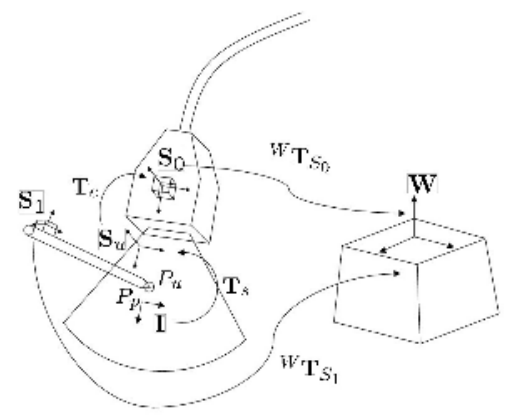

(a)

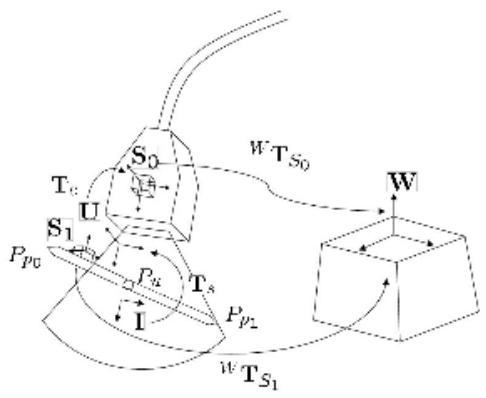

(b)

Fig. 1. (a) depicts the calibration method, which requires exact placement of the pointer tip in the ultrasonic plane. (b) shows our new method that only requires intersection anywhere along the pointer.

image is poorly resolved. Furthermore, the width (elevational thickness) of the ultrasound beam is not infinitesimally small, the lower bound of the error in determining the position of the pointer tip is half of the beam width.

\subsection{Proposed Phantom-Less Calibration Method}

The proposed phantom-less calibration method addresses the problem of the method discussed in section 2.1. Two or more points on the calibration stylus (pointer) are assumed to be known. In this way, the orientation of the calibration pointer is known. We denote the coordinates of these points as $P_{p_{0}}$ and $P_{p_{1}}$ in the coordinate system of the tracker sensor $\mathbf{S}_{1}$ attached to the pointer. If we position the stylus in a way that it intersects the ultrasound beam, we have the following equation:

$$
{ }^{W} \mathbf{T}_{S_{1}}\left(P_{p_{0}}+\lambda \frac{P_{p_{1}}-P_{p_{0}}}{\left|P_{p_{1}}-P_{p_{0}}\right|}\right)={ }^{W} \mathbf{T}_{S_{0}} \mathbf{T}_{c} \mathbf{T}_{s} P_{u},
$$

where $\lambda$ is an unknown real number with in $\left[\begin{array}{ll}0 & 1\end{array}\right]$. In order to omit the unknown factor $\lambda$, equation 4 can e re-written as follows:

$$
\left[L_{01}\right]_{x}\left[{ }^{W} \mathbf{T}_{S_{0}} \mathbf{T}_{c} \mathbf{T}_{s} P_{u}-{ }^{W} \mathbf{T}_{S_{1}} P_{p_{0}}\right]_{3}=\mathbf{0}_{3 \times 1},
$$

where the operator $[.]_{x}$ converts a vector to a skew symmetric matrix, and $\mathbf{0}_{3 \times 1}$ represents a null point. Furthermore, $L_{01}$ is the normalized vector within the world coordinate system, which is connecting the points $P_{p_{0}}$ and $P_{p_{1}}$ with $L_{01}=$ $\frac{{ }^{W} \mathbf{R}_{S_{1}}\left[P_{p_{1}}\right]_{3}-{ }^{W} \mathbf{R}_{S_{1}}\left[P_{p_{0}}\right]_{3}}{\left\|\left[P_{p_{1}}-P_{p_{0}}\right]_{3}\right\|} .{ }^{W} \mathbf{R}_{S_{1}}$ is the $3 \times 3$ rotation matrix imbedded in ${ }^{W} \mathbf{T}_{S_{1}}$. Finally if the measurements are done for various points $i$, a similar relationship as in equation (3) can be established to solve for calibration parameters: 


$$
\left\{\widetilde{\mathbf{T}_{\mathbf{c}}}, \widetilde{\mathbf{T}_{s}}\right\}=\arg \min _{\left[\mathbf{T}_{c}, \mathbf{T}_{s}\right]} \sum_{i}\left\|\left[L_{01}^{i}\right]_{x}\left[{ }^{W} \mathbf{T}_{S_{0}}^{i} \mathbf{T}_{c} \mathbf{T}_{s} P_{u}^{i}-{ }^{W} \mathbf{T}_{S_{1}} P_{p_{0}}\right]_{3}\right\|^{2}
$$

The difference between equations (3) and (6) is the matrix $\left[L_{01}^{i}\right]_{x}$, which specifies the direction of the pointer and relaxes the constraint of exact intersection of the pointer tip with the ultrasound plane.

\subsection{Calibration Workflow}

The calibration can be divided into three processes. First is the pointer calibration, during which the coordinates of two points along the stylus have to be determined accurately within the coordinate system of the sensor attached to the stylus. In the second step, the spatial calibration acquisition is done. The pointer is placed into multiple positions within the ultrasonic beam, preferably using a grid guide that is well spread over the ultrasound image plane. The 6DOF sensor readings along with the $2 \mathrm{D}$ coordinates of the intersection points on the image is recorded and used for recovering the spatial calibration parameters. The third process is for temporal calibration. During this step, we continuously move the pointer while keeping it intersecting the ultrasound plane. We record 2D coordinates of the pointer trace within the ultrasound image, 6DOF coordinates of the pointer sensor,and the corresponding time stamps. These recordings are then used to recover the (semi) constant delay between the magnetic pose and the ultrasound image acquisition.

Pointer Calibration. Pointer tip calibration can be performed by fixing the tip of the pointer and rotating the pointer about the fixed point. For two distinctive poses of the pointer (e.g., ${ }^{W} \mathbf{T}_{S_{1}}^{0}$ and ${ }^{W} \mathbf{T}_{S_{1}}^{1}$ ), we have ${ }^{W} \mathbf{T}_{S_{1}}^{0} P_{p}={ }^{W} \mathbf{T}_{S_{1}}^{1} P_{p}$. Therefore, the pointer tip is simply:

$$
\left[P_{p}\right]_{3}=\left({ }^{W} \mathbf{R}_{S_{1}}^{0}-{ }^{W} \mathbf{R}_{S_{1}}^{1}\right)^{-1}\left({ }^{W} \mathbf{t}_{S_{1}}^{0}-{ }^{W} \mathbf{t}_{S_{1}}^{1}\right) .
$$

where $\mathbf{R}$ is the rotation matrix and $\mathbf{t}$ is the translation vector embedded in homogenous transformation matrix $\mathbf{T}$. For more robust and accurate solution and to avoid degeneracy in equation 7 more than two poses (e.g., $n$ ) should be used. In this case, we select any combination pair of points, and solve a least squares problem using matrix manipulations, as follows:

$$
\left[P_{p}\right]_{3}=\left(\mathbf{R R}^{\top} \mathbf{R R}\right)^{-1} \mathbf{R R}^{\top} \mathbf{t t}
$$

where $\mathbf{R R}=\left[\left.\ldots\right|^{W} \mathbf{R}_{S_{1}}^{i}-{ }^{W} \mathbf{R}_{S_{1}}^{j} \mid \ldots\right]$, and $\mathbf{t t}=\left[\ldots\left|{ }^{W} \mathbf{t}_{S_{1}}^{i}-{ }^{W} \mathbf{t}_{S_{1}}^{j}\right| \ldots\right]$, and $(i, j)$ is a combination pair from $[1, n]$.

Spatial Calibration. For the spatial calibration, we first overlay an equidistant grid of points onto the ultrasound image plane. The user's task is to intersect the pointer with the ultrasound plane in a way that the grid points are lined up with the trace of the pointer in the image. In order to avoid degeneracy in the solution of equation (6) , it is required the user change the orientation of the pointer from one grid point to another. At each grid point, the coordinates 
Table 1. (a) Pointer calibration results, (b) extracted spatial calibration parameters

\begin{tabular}{|l|c|c|c|}
\hline Point & $\mathrm{x}$ & $\mathrm{y}$ & $\mathrm{z}$ \\
\hline$P_{p_{0}}(\mathrm{~mm})$ & -58.644 & -1.1899 & -11.371 \\
$P_{p_{1}}(\mathrm{~mm})$ & 34.800 & 6.6530 & -9.1214 \\
\hline
\end{tabular}

(a)

\begin{tabular}{|l|c|c|c|}
\hline Calib. & $\mathrm{x}$ & $\mathrm{y}$ & $\mathrm{z}$ \\
\hline Trans. (mm) & 27.891 & 21.967 & -62.082 \\
Rot. (deg) & -47.038 & -70.526 & -44.386 \\
Scale & 0.187 & 0.196 & - \\
\hline
\end{tabular}

(b)

of the grid and the pose of the sensors attached to the pointer and ultrasound probe are saved. It is desirable that the user performs the acquisition in the vicinity of the workspace, where the final measurements are done specially in the case, where magnetic trackers are used. This ensures that magnetic tracker readings are as consistent as possible. Furthermore, it is better to change the pose of the ultrasound probe as the user select different grid point to account for possible variations in magnetic readings and minimize the bias in the results by perturbing the measurement error.

Temporal Calibration. We assume that tracker and ultrasound acquisition frequencies are known. What remains unknown is the inherent delay that exists between the two processes. In order to measure this delay, the user holds the pointer intersecting the ultrasound plane in a perpendicular fashion. The user should periodically move the pointer in horizontal or vertical direction back and forth, while the ultrasound probe is steady. During the motion, both the ultrasound images and the pose of the pointer sensor are recorded together with the time stamps. We then process the recorded images and extract the bright points (in pixels) representing the pointer intersections. The dominant direction of the point in the image (in pixels) and dominant translation parameter of the sensor (in millimeters) are then used to recover the delay. Let us assume the dominant direction of image point movement is in horizontal direction (i.e. $r(t)$, where $t$ is time) and the dominate translation is in $x$ direction (i.e., $x(t)$ ). The delay can be computed using the following equation:

$$
\tau=\arg \max \left(\mathbf{F}^{-1}\left\{\frac{\mathbf{F}\{r(t)\} \mathbf{F}^{*}\{x(t)\}}{\left|\mathbf{F}\{r(t)\} \mathbf{F}^{*}\{x(t)\}\right|}\right\}\right)
$$

where $\mathbf{F}$ and $\mathbf{F}^{-1}$ represent Fourier and inverse Fourier transforms, respectively, and $(*)$ denotes the complex conjugate operation.

\section{Experimental Results}

We performed a series of tests in order to verify the performance of the proposed calibration process. Performance measures are considered to be the amount of residual error in the optimization process for the calibration parameters and the quality of three dimensional compounding of the tracked B-plane images. In this experiments, we used images acquired by a SONOLINE Elegra ultrasound (Siemens Medical Solutions, Issaquah, WA) with a 50HDPL40 probe. As tracking device, we used a MicroBird magnetic tracking system (Ascension, 


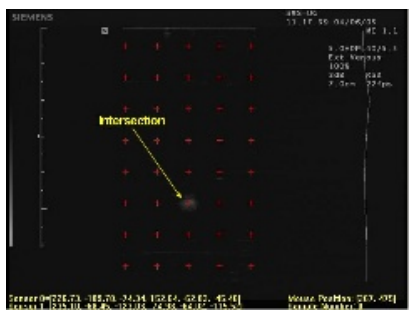

(a)

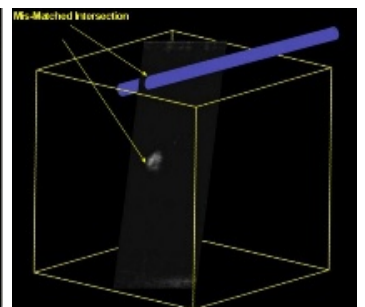

(b)

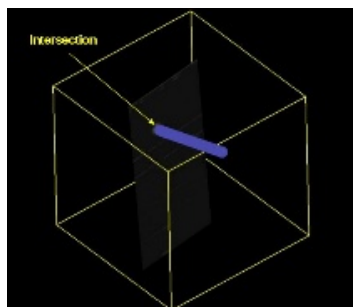

(c)

Fig. 2. (a) depicts the ultrasound image with projected grid pattern, (b) shows the discrepancy between the pointer intersection according to the tracking system and its trace in the image, (c) the discrepancy is minimized after the calibration process.

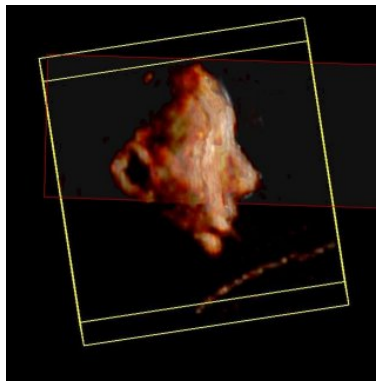

(a)

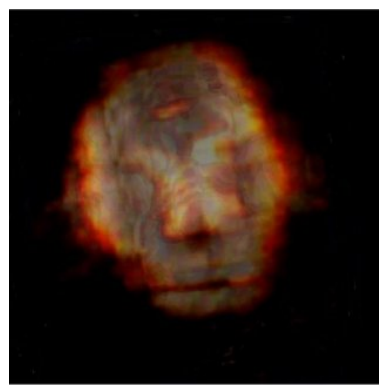

(b)

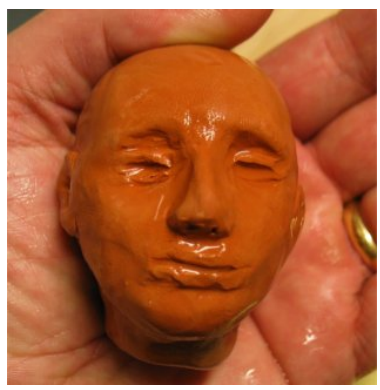

(c)

Fig. 3. (a) depicts the compounded volume with overlaid B-plane, (b) shows the volume from another view and (c) is the photograph of the actual clay model.

Burlington, VT). We prepared a $10 \mathrm{~cm}$ stainless steel rod as the pointer. We attached a magnetic sensor to the middle of the rod ,sharpened and calibrated both ends using the method described in section 2.3. The standard deviations of the end points in the world coordinate system were $0.9501 \mathrm{~mm}$ and $0.6068 \mathrm{~mm}$. The estimated coordinates of the end points are listed in Table1(a). We acquired calibration data as explained in section 2.3. By assuming nominal scaling for the ultrasound image, and relaxing the orthonormality constraints of the rotation matrix, we found an estimate of the calibration parameters in a closed-form fashion using various measurements through equation (5). The results were then used as initial values for the optimization in equation (6). We used LevenbergMarquardt method to solve the non-linear optimization problem. The residual error of the optimization process was $0.8913 \mathrm{~mm}$. Figure2 (b) shows the intersection of the virtual pointer and the ultrasound plane with arbitrary calibration parameters. There is a clear discrepancy between the virtual and real intersections. Figure 2 (c) shows a good match after computing the correct calibration parameters. The temporal calibration delay was computed to be about 96 milliseconds. The refresh rates of the magnetic tracker and ultrasound device were 60 and 30 $\mathrm{Hz}$, respectively. Since the frequencies were not synchronized up to 16 millisecond 
variable time discrepancy exists between image and tracked pose. Figures 3 (a) and (b) show reconstruction of the clay head, depicted in Figure 3 (c), using the real-time compounding method described in 4. The reconstruction is performed by sweeping the B-plane axially from head to neck. Figure 3 (a) shows the reconstruction overlaid by a B-plane in sagittal orientation. Matching outlines in B-plane and reconstruction confirm the fidelity of the calibration parameters.

\section{Summary and Conclusion}

Calibration is essential for a tracked ultrasound system. In this paper, we propose a robust phantom-less calibration approach. With this method, one can extract both spatial and temporal calibration parameters in a unified way. During spatial calibration, no image processing is needed. Some image processing is required for temporal calibration. Main advantage of the method is minimizing the user dependency during the acquisition of the data for spatial calibration. The new approach relies on the orientation of the tracked pointer instead of the exact tip position. Reconstruction of clay model illustrates the quality of the calibration.

\section{References}

1. Barry, C. D., Allot, C. P., John, N. W., Mellor, P. M., Arundel, P. A., Thomson, D. S. and Waterton, J., Three-dimensional freehand ultrasound: image reconstruction and volume analysis. Ultrasound in Med. and Biol. 23(8), 1997, 1209-1224.

2. Bouchet, L. G., Meeks, S. L., Goodchild, G., Bova, F. J., Buatti, J. M., and Friedman W. A., Calibration of three-dimensional ultrasound images for image guided radiation therapy, Physics in Med. and Biol., 46, 2001, 559-577.

3. Detmer, P. R., Bashin, G., Hodges, T., Beach K. W., Filter, E. P., Burns, D. H., Strandness Jr., D. E., 3D Ultrasound image feature localization based on magnetic scanhead tracking: in vitro calibration and validation, Ultrasound in Med. and Biol., 20(9), 1994, 923-936.

4. -

5. Muratore, D. M. , and Galloway, Jr, R. L.,Beam calibration without a phantom for creating a 3D free-hand ultrasound system, Ultrasound in Med. and Biol., 27(11), 2001, 1557-1566.

6. Pagoulatos, N, Haynor, D. R., and Kim, Y.,A Fast calibration method for 3D tracking of ultrasound images using a spatial localizer, Ultrasound in Med. and Biol., 27(9), 2001, 1219-1229.

7. Prager, R.W., Rohling, R. N., Gee, A. N., Berman, L., Rapid calibration for 3-D freehand ultrasound, Ultrasound in Med. and Biol. 24(6),1998, 855-869.

8. Prager, R.W., Gee, A. N., Berman, L., StradX: Real-time acquisition and visulization of freehand three-dimensional ultrasound, Med. Image Anal. 3(2),1999, 129-140.

9. Sato, Y., Nakamoto, M., Tamaki, Y., Sasama, T., Sakita, I., Nakajima, Y., Monden, M., Tamura, S., Image guidance of breast cancer surgery using 3-D ultrasound images and augmented reality visualization, IEEE Trans. on Med. Imag. 17(5), 1998, 681-693. 